PROCEEDINGS OF THE

AMERICAN MATHEMATICAL SOCIETY

Volume 128, Number 6, Pages 1633-1639

S 0002-9939(99)05234-X

Article electronically published on October 27, 1999

\title{
A CHARACTERIZATION OF TOTAL REFLECTION ORDERS
}

\author{
PAOLA CELLINI
}

(Communicated by Ronald M. Solomon)

\begin{abstract}
Let $(W, S)$ be a Coxeter system with set of reflections $T$. It is known that if $\prec$ is a total reflection order for $W$, then, for each $s \in S,\{t \in$ $T \mid t \prec s\}$ and its complement are stable under conjugation by $s$. Moreover the upper and lower $s$-conjugates of $\prec$ are still total reflection orders. For any total order $\prec$ on $T$, say that $\prec$ is stable if $\{t \in T \mid t \prec s\}$ is stable under conjugation by $s$ for each $s \in S$. We prove that if $\prec$ and all orders obtained from $\prec$ by successive lower or upper $S$-conjugations are stable, then $\prec$ is a total reflection order.
\end{abstract}

\section{INTRODUCTION}

The notion of total reflection order was introduced by Dyer [D2] in the context of his study of reflection subgroups in Coxeter systems and its further developments regarding Hecke algebras and the related Kazhdan-Lusztig theory. The original definition is introduced in terms of dihedral reflection subgroups and has the following equivalent geometric formulation [D2]. If $(W, S)$ is a Coxeter system, $T$ is the set of its reflections, and $\Phi$ is its root system in the geometric representation $[\underline{H}]$, then there is a natural bijection between the set $\Phi^{+}$of positive roots and $T$, which maps the root $\alpha \in \Phi^{+}$to the reflection $r_{\alpha}$ in $T$. A total reflection order is a total order $\prec$ on $T$ which satisfies the following convexity condition: for each $\alpha, \beta \in \Phi^{+}$and $c, d \in \mathbb{R}^{+}$such that $c \alpha+d \beta \in \Phi^{+}$, either $r_{\alpha} \prec r_{c \alpha+d \beta} \prec r_{\beta}$ or $r_{\beta} \prec r_{c \alpha+d \beta} \prec r_{\alpha}$.

This notion has interesting applications: for instance, Dyer [D2 and Brenti [B1], [B2] give respectively formulas for the $R$-polynomials and the Kazhdan-Lusztig polynomials involving total reflection orders.

If $\prec$ is a total reflection order, then, for each $s \in S$, the sets $\{t \in T \mid t \prec s\}$ and $\{t \in T \mid t \succ s\}$ are stable under conjugation by $s$ [D2]. We shall call stable any total order on $T$ that satisfies this property. The upper $s$-conjugate of a stable order $\prec$ is the total order $\prec^{s}$ on $T$ defined as follows: for each $t, t^{\prime} \in T, t \neq t^{\prime}$, we have $t \prec^{s} t^{\prime}$ if and only if either $t \prec t^{\prime} \prec s$, or $t \prec s \prec t^{\prime}$, or $s \prec s t s \prec s t^{\prime} s$, or $t^{\prime}=s$. The lower $s$-conjugate $\prec_{s}$ of $\prec$ is defined similarly. Dyer [D2] proves that if $\prec$ is a total reflection order, then $\prec_{s}$ and $\prec^{s}$ are total reflection orders too; thus they are stable. Stability and preservation of stability under successive upper or lower $S$-conjugations are exactly the properties of total reflection orders which are needed for the explicit computation of the $R$-polynomials and hence of Kazhdan-Lusztig polynomials (see $[\mathrm{BB}, 8]$ ). We say that a total order on $T$ is a $K L$-order if it is

Received by the editors July 30, 1998.

1991 Mathematics Subject Classification. Primary 20F55; Secondary 05E99.

Key words and phrases. Coxeter groups, total reflection orders. 
stable and each successive upper or lower $S$-conjugate of it is stable. Indeed, as is seen in the proof of Dyer's formulas for the $R$-polynomials given in BB 8.4], such formulas hold for any fixed $K L$-order. For this reason, we investigate these orders. By Dyer's results any total reflection order is $K L$. The main result of this paper is that the converse holds too.

Theorem 1.1. Any KL-order is a total reflection order.

Thus the $K L$-property characterizes the total reflection orders.

Theorem 1.1 is proved in section 3 . In section 4 we prove that, if $W$ is finite, for a total order on $T$ to be $K L$ it is sufficient to be stable under successive just upper or just lower $S$-conjugations. Moreover, we prove that if each proper parabolic subgroup of $W$ is finite, then the stable orders on $T$ which remain stable under successive upper (lower) $S$-conjugations have the same finite initial (final) sections as those of total reflection orders.

\section{NotATION AND PRELIMINARIES}

In this section we fix notation and recall some well known facts (see [De] or [H] Chapter 5]). Let $(W, S)$ be a Coxeter system. Then $T=\left\{w s w^{-1} \mid w \in W, s \in S\right\}$ is the set of reflections of $W$. It is well known that $W$ can be realized as a real reflection group as follows. Let $V$ be a real vector space of dimension $|S|$ with basis $\Pi=\left\{\alpha_{s} \mid s \in S\right\}$, and define the standard bilinear form $(\cdot, \cdot)$ on $V$ as the unique symmetric bilinear form such that $\left(\alpha_{r}, \alpha_{s}\right)=-\cos \frac{\pi}{m(r, s)}$ for each $r, s \in S$, where $m(r, s)$ is the order of $r s$. For a non-isotropic $\alpha \in V$ let $r_{\alpha}$ denote the reflection in $\alpha, r_{\alpha}(x)=x-\frac{2(\alpha, x)}{(\alpha, \alpha)} \alpha$, for each $x \in V$. Then the map $s \mapsto r_{\alpha_{s}}$, for $s \in S$, extends to a faithful representation of $W$ in $G L(V)$, the geometric representation, so that $W$ is isomorphic to the reflection group $\left\langle r_{\alpha_{s}} \mid s \in S\right\rangle$. We identify $W$ with such a reflection group and $s$ with $r_{\alpha_{s}}$, for each $s \in S$. The standard bilinear form is $W$ invariant. The root system of $W$ is defined as $\Phi=W \Pi=\{w(\beta) \mid w \in W, \beta \in \Pi\}$; a root $\alpha \in \Phi$ is called positive $(\alpha>0)$ if $\alpha=\sum_{\beta \in \Pi} c_{\beta} \beta$ with $c_{\beta} \geq 0$, and $\Phi^{+}$ denotes the set of all positive roots. Then $\Phi=\Phi^{+} \cup-\Phi^{+}$; if $\alpha \in-\Phi^{+}$, then $\alpha$ is called negative $(\alpha<0)$. We have $r_{\beta}=r_{-\beta}$ and $r_{w(\beta)}=w r_{\beta} w^{-1}$, for each $\beta \in \Phi$ and $w \in W$, so that $T=\left\{r_{\alpha} \mid \alpha \in \Phi^{+}\right\}$and the map $\alpha \mapsto r_{\alpha}$ is a bijection between $\Phi^{+}$and $T$. Moreover each element of $W$ which acts as a reflection on $V$ belongs to $T$ [H, 5.8, Exercise 3].

For each $w \in W$ set $N(w)=\{t \in T \mid \ell(t w)<\ell(w)\}$, where $\ell$ is the length function of $(W, S)$. Then we have $|N(w)|=\ell(w)$; moreover if $t=r_{\alpha}$ with $\alpha \in \Phi^{+}$, then $\ell(t w)<\ell(w)$ if and only if $w^{-1}(\alpha)<0$. Thus it is easy to see that, for each $v, w \in W$, we have $N(v w)=N(v)+v N(w) v^{-1}$, where + denotes the symmetric difference of sets.

A subgroup $G$ of $W$ is called a reflection subgroup if $G=\langle G \cap T\rangle$. For any reflection subgroup $G$ of $W$, the set $\chi(G)=\{t \in T \mid N(t) \cap G=\{t\}\}$ is a set of Coxeter generators for $G$ [De], D1]. Moreover, if $T_{G}=\left\{g t g^{-1} \mid g \in G, t \in \chi(G)\right\}$ is the set of reflections of $(G, \chi(G)), \ell_{G}$ is the length function, and $N_{G}(v)=\{t \in$ $\left.T_{G} \mid \ell_{G}(t v)<\ell_{G}(v)\right\}$ for each $v \in G$, then $T_{G}=T \cap G$ and $N_{G}(v)=N(v) \cap G$, for each $v \in G$ [D1, 3.3].

A reflection subgroup $G$ is called dihedral if $|\chi(G)|=2$. If $D$ is a dihedral reflection subgroup with $\chi(D)=\{r, t\}$, we set $r_{i}=(r t)^{i} r$, and $t_{i}=(t r)^{i} t$, for $i \in \mathbb{N}$, so that $T_{D}=\left\{r_{i}, t_{i} \mid i \geq 0\right\}$. 
Definition 2.1 ( $[\overline{\mathrm{D}} 2]$ ). A total reflection order of $W$ is a total order $\prec$ on $T$ which satisfies the following condition: for each dihedral reflection subgroup $D$ of $W$, if $\chi(D)=\{r, t\}$ and $r \prec t$, then

$$
r_{i-1} \prec r_{i} \preccurlyeq t_{i} \prec t_{i-1}
$$

for $1 \leq i<\frac{m(r, t)}{2}$, where $m(r, t)$ is the order of $r t$.

The definition can be geometrically reformulated as follows D2. Let $\prec$ be a total order on $T$ and let $\prec^{\prime}$ be the order induced on $\Phi^{+}$by the natural bijection. Then $\prec$ is a total reflection order if and only if, for each $\alpha, \beta \in \Phi^{+}$and $c, d \in \mathbb{R}^{+}$ such that $c \alpha+d \beta \in \Phi^{+}$, if $\alpha \prec^{\prime} \beta$, then $\alpha \prec^{\prime} c \alpha+d \beta \prec^{\prime} \beta$.

Henceforth we denote by · the standard left action of $W$ on its subsets given by conjugation; thus for $A \subseteq W$ and $w \in W, w \cdot A=w A w^{-1}$.

Let $\prec$ be a total order on $T$. For each $t \in T$ we set $(\prec t)=\left\{t^{\prime} \in T \mid t^{\prime} \prec t\right\}$ and $(\succ t)=\left\{t^{\prime} \in T \mid t^{\prime} \succ t\right\}$. We say that $\prec$ is stable if, for each $s \in S, s \cdot(\prec s)=(\prec s)$; in such a case we also have $s \cdot(\succ s)=(\succ s)$. Let $\prec$ be stable. Then, for $s \in S$, the upper $s$-conjugate $\prec^{s}$ of $\prec$ is defined as follows:

$$
t \preccurlyeq^{s} s \quad \text { for each } t \in T ; \quad t \prec^{s} t^{\prime} \quad \text { if } \quad\left\{\begin{array}{l}
t \prec t^{\prime} \prec s, \quad \text { or } \\
t \prec s \prec t^{\prime}, \quad \text { or } \\
s \prec s t s \prec s t^{\prime} s .
\end{array}\right.
$$

Thus $\prec^{s}$ is obtained from $\prec$ by shifting $s$ to the last position and conjugating by $s$ each overtaken element. The lower $s$-conjugate $\prec_{s}$ of $\prec$ is defined similarly: $s \preccurlyeq s t$ for each $t \in T$; $t \prec_{s} t^{\prime}$ if either sts $\prec s t^{\prime} s \prec s$, or $t \prec s \prec t^{\prime}$, or $s \prec t \prec t^{\prime}$. The following result holds:

Proposition ([D2, 2.4-2.5]). If $\prec$ is a total reflection order, then $\prec$ is stable and, for each $s \in S, \prec^{s}$ and $\prec_{s}$ are total reflection orders.

If $\prec$ and $\prec^{\prime}$ are total orders on $T$, we set $\prec \longrightarrow \prec^{\prime}$ if $\prec$ is stable and either $\prec^{\prime}=\prec_{s}$ or $\prec^{\prime}=\prec^{s}$ for some $s \in S$. We define $\rightarrow$ as the transitive closure of $\rightarrow$. Thus $\prec \rightarrow \prec^{\prime}$ if and only if $\prec^{\prime}$ is obtained from $\prec$ by successive lower or upper $S$-conjugations, each conjugation being performed on a stable order.

Definition 2.2. Let $\prec$ be a total order on $T$. We say that $\prec$ is a $K L$-order if $\prec$ is stable and each total order $\prec^{\prime}$ such that $\prec \rightarrow \prec^{\prime}$ is stable.

By the above Proposition any total reflection order is $K L$. We remark that the explicit formulas for the $R$-polynomials and $K L$-polynomials of $W$ given in D2, [B1], B2] in terms of a total reflection order hold with a $K L$-order. So it is natural to investigate whether the $K L$ property is weaker than that of being a total reflection order. In the next section we prove that indeed the $K L$ property characterizes the total reflection orders.

\section{Proof of Theorem 1.1}

Henceforth we denote by $D$ a dihedral subgroup of $W$ with $\chi(D)=\{r, t\}$, and by $m$ the order of $r t$. Moreover we denote by $\prec$ a fixed $K L$-order. In this section we shall prove that $\prec$ satisfies the condition of Definition 2.1. For $m=2$ the condition is void, so we assume $m>2$, possibly $m=\infty$. We keep in force the notation fixed above Definition 2.1. Note that if $D$ is finite, then we have $T_{D}=\left\{r_{i} \mid 0 \leq i<m\right\}$. 
Lemma 3.1. Let $x, y, z \in T_{D}$ and $x \prec y \prec z$. Then there exist a KL-order $\prec^{\prime}$ on $T$ and an element $w \in W$ such that $\chi\left(D^{w}\right)=\left\{r^{w}, t^{w}\right\}, r^{w}$ is simple, and $x^{w} \prec^{\prime} y^{w} \prec^{\prime} z^{w}$.

Proof. Let $r=s_{1} \cdots s_{k} s s_{k} \cdots s_{1}$, with $s \in S, s_{i} \in S$ for $i=1, \ldots, k$, and $\ell(r)=$ $2 k+1$ (for the existence of such a reduced expression see [H, 5.8, Exercise 3]). Set $w_{0}=1$, and $w_{i}=s_{1} \cdots s_{i}$, for $i=1, \ldots, k$. First we notice that for each $u \in T_{D}$ and $0 \leq i<k$ we have $u^{w_{i}} \neq s_{i+1}$. Otherwise, we would have $u=s_{1} \cdots s_{i} s_{i+1} s_{i} \cdots s_{1}$ and thus $u r=s_{1} \cdots \widehat{s_{i+1}} \cdots s_{k} s s_{k} \cdots s_{1}$, where "^, denotes omission. Thus $\ell(u r)<$ $\ell(r)$, but $N(r) \cap T_{D}=\{r\}$ and $u \neq r$ : a contradiction. Therefore we have that $s_{i+1} \notin D^{w_{i}}$ for $i=0, \ldots, k-1$. By [D1, 3.2(i)], it follows that $\chi\left(D^{s_{1}}\right)=\left\{r^{s_{1}}, t^{s_{1}}\right\}$ and, inductively, that $\chi\left(D^{w_{i}}\right)=\left\{r^{w_{i}}, t^{w_{i}}\right\}$, for $i=0, \ldots, k$.

Now set $\prec_{0}=\prec$, let $0 \leq i<k$, and assume that we have defined a $K L$-order $\prec_{i}$ on $T$ such that $x^{w_{i}} \prec_{i} y^{w_{i}} \prec_{i} z^{w_{i}}$. Then we define a $K L$-order $\prec_{i+1}$ as follows. If either $s_{i+1} \prec_{i} x^{w_{i}} \prec_{i} y^{w_{i}} \prec_{i} z^{w_{i}}$, or $x^{w_{i}} \prec_{i} s_{i+1} \prec_{i} y^{w_{i}} \prec_{i} z^{w_{i}}$, then we define $\prec_{i+1}$ as the upper conjugate $\left(\prec_{i}\right)^{s_{i+1}}$. If either $x^{w_{i}} \prec_{i} y^{w_{i}} \prec_{i} z^{w_{i}} \prec_{i} s_{i+1}$, or $x^{w_{i}} \prec_{i} y^{w_{i}} \prec_{i} s_{i+1} \prec_{i} z^{w_{i}}$, then we define $\prec_{i+1}$ as the lower conjugate $\left(\prec_{i}\right)_{s_{i+1}}$. Then it is easily seen that $x^{w_{i+1}} \prec_{i+1} y^{w_{i+1}} \prec_{i+1} z^{w_{i+1}}$. Thus $w=w_{k}$ and $\prec^{\prime}=\prec_{k}$ have the claimed properties.

Proposition 3.2. If $r \prec t$, then $r \prec r t r$ and $t r t \prec t$.

Proof. Assume by contradiction that $r t r \prec r \prec t$. By Lemma 3.1 we can find a dihedral subgroup $D^{\prime}$, with $\chi\left(D^{\prime}\right)=\left\{r^{\prime}, t^{\prime}\right\}, r^{\prime}$ simple, and a $K L$-order $\prec^{\prime}$ such that $r^{\prime} t^{\prime} r^{\prime} \prec^{\prime} r^{\prime} \prec^{\prime} t^{\prime}$. This is a contradiction since $\left(r^{\prime} \prec^{\prime}\right)$ must be stable under conjugation by $r^{\prime}$. Hence $r \prec r t r$ as claimed. Similarly we have $t r t \prec t$.

Lemma 3.3. If $r \prec t$, then $r_{i-1} \prec r_{i}$ and $t_{i} \prec t_{i-1}$ for $1 \leq i<m$.

Proof. We prove the claim by induction. Let $1 \leq k<m-1$ and assume that for each $K L$-order $\prec^{\prime}$ and for each dihedral subgroup $D^{\prime}$ with $\chi\left(D^{\prime}\right)=\left\{r^{\prime}, t^{\prime}\right\}$, if $r^{\prime} \prec^{\prime} t^{\prime}$, then we have $r_{i-1}^{\prime} \prec^{\prime} r_{i}^{\prime}$ and $t_{i}^{\prime} \prec^{\prime} t_{i-1}^{\prime}$ for $1 \leq i \leq k$. For $k=1$ this follows from Proposition 3.2. Assume by contradiction that $r_{k+1} \prec r_{k}$. By Lemma 3.1 we can find an element $w \in W$ and a $K L$-order $\prec^{\prime}$ such that $r^{\prime}=r^{w}$ is simple, $r^{\prime} \prec^{\prime} r_{k}^{\prime}$, and $r_{k+1}^{\prime} \prec^{\prime} r_{k}^{\prime}$, where $u^{\prime}=u^{w}$ for each $u$. Since $r^{\prime} \prec^{\prime} r_{k}^{\prime}$, by the inductive assumption we have $r^{\prime} \prec^{\prime} t^{\prime}$ and $r^{\prime} \prec^{\prime} r_{1}^{\prime} \preccurlyeq^{\prime} \cdots \preccurlyeq^{\prime} r_{k}^{\prime}$. Now we consider the upper conjugate $\prec^{\prime \prime}=\left(\prec^{\prime}\right)^{r^{\prime}}$. Since $\left(r_{i}^{\prime}\right)^{r^{\prime}}=t_{i-1}^{\prime}$, we obtain $t^{\prime} \preccurlyeq^{\prime \prime} t_{k-1}^{\prime}$; hence, by the inductive assumption, $t^{\prime} \preccurlyeq^{\prime \prime} t_{k-1}^{\prime} \prec^{\prime \prime} t_{k}^{\prime} \prec^{\prime \prime} r^{\prime}$. But we have $r_{k+1}^{\prime} \prec^{\prime} r_{k}^{\prime}$, $r^{\prime} \prec^{\prime} r_{k}^{\prime}$, and $r^{\prime} \neq r_{k+1}^{\prime}$; hence $t_{k}^{\prime} \prec^{\prime \prime} t_{k-1}^{\prime}$, a contradiction. Therefore the claim is proved.

Proposition 3.4. If $D$ is finite and $r \prec t$, then $r \prec r_{1} \prec \cdots \prec r_{m-1}=t$. If $D$ is infinite and $r \prec t$, then $r_{i} \prec r_{i+1} \prec t_{j+1} \prec t_{j}$ for each $i$ and $j \geq 0$.

Proof. If $D$ is finite, the claim follows directly from Lemma 3.3. Assume that $D$ is infinite. By Lemma 3.3 we have only to prove that $r_{i} \prec t_{j}$ for each $i, j \geq 0$.

First we prove that, for each $i, j \geq 0$, the subgroup $D_{i j}=\left\langle r_{i}, t_{j}\right\rangle$ is dihedral and $\chi\left(D_{i j}\right)=\left\{r_{i}, t_{j}\right\}$. For $k \geq 1$, we have $N_{D}\left(r_{i+1}\right)=N_{D}\left(r_{i} t r\right)=N_{D}\left(r_{i}\right)+r_{i} \cdot N_{D}(t)+$ $r_{i} t \cdot N_{D}(r)=N_{D}\left(r_{i}\right)+\left\{r_{i} t r_{i}\right\}+\left\{r_{i} t r t r_{i}\right\}=N_{D}\left(r_{i}\right)+\left\{r_{2 i+1}, r_{2 i+2}\right\}$. Since $r_{0}=r$, we have $N_{D}\left(r_{0}\right)=\left\{r_{0}\right\}$ and hence, by induction, $N_{D}\left(r_{i}\right)=\left\{r_{h} \mid 0 \leq h \leq 2 i\right\}$, for each $i \geq 0$. Similarly we have $N_{D}\left(t_{j}\right)=\left\{t_{k} \mid 0 \leq k \leq 2 j\right\}$, for each $j \geq 0$. Now we 
have $r_{i} t_{j} r_{i}=r_{2 i+j+1}$; therefore, if $r_{h} \in D_{i j}$ and $r_{h} \neq r_{i}$, then $h>2 i$. It follows that $N\left(r_{i}\right) \cap D_{i j}=N_{D}\left(r_{i}\right) \cap D_{i j}=\left\{r_{i}\right\}$. Similarly, we have $N\left(t_{j}\right) \cap D_{i j}=\left\{t_{j}\right\}$.

Now if, by contradiction, $t_{j} \prec r_{i}$ for some $i$ and $j$, then, applying Lemma 3.3 to the dihedral subgroup $D_{i j}$, we would obtain $t_{j} \prec t_{j} r_{i} t_{j}=t_{2 j+i+1}$. But by Lemma 3.3 applied to $D$, we have $t_{2 j+i+1} \prec t_{j}$. Thus we conclude that $r_{i} \prec t_{j}$ for all $i, j \geq 0$.

Proposition 3.4 shows that $\prec$ satisfies the conditions of Definition 2.1; thus the proof is complete.

\section{ONE SIDE STABILITY}

In this section we consider stability under successive one side $S$-conjugations.

Definition 4.1. Let $\prec$ be a total order on $T$. If $\prec$ and $\prec^{\prime}$ are total orders on $T$, we set $\prec \stackrel{u}{\rightarrow} \prec^{\prime}$ if $\prec$ is stable and $\prec^{\prime}=\prec^{s}$ for some $s \in S$. We define $\stackrel{u}{\rightarrow}$ as the transitive closure of $\stackrel{u}{\rightarrow}$. We say that a total order $\prec$ on $T$ is $u$-stable if $\prec$ and each $\prec^{\prime}$ such that $\prec \stackrel{u}{\rightarrow} \prec^{\prime}$ are stable.

Similarly, we define the $l$-stable orders of $T$ as the stable orders which remain stable under successive lower conjugations. Clearly, a total order $\prec$ on $T$ is $l$-stable if and only if the reverse order is $u$-stable.

We recall that if $w=s_{1} \cdots s_{k}$ is reduced, then we have $N(w)=\left\{t_{1}, \ldots, t_{k}\right\}$, where $t_{1}=s_{1}, t_{i}=s_{1} \ldots s_{i-1} s_{i} s_{i-1} \cdots s_{1}$; moreover $w=t_{k} \cdots t_{1}$. With this notation, we say that the order $t_{1} \prec \cdots \prec t_{k}$ is induced by the reduced expression $s_{1} \cdots s_{k}$ of $w$. By [D2, 2.11], $A \subseteq T$ is a finite initial section of some total reflection order if and only if $A=N(w)$ for some $w \in W$. Moreover, $t_{1} \prec \cdots \prec t_{k}$ is a finite initial section of $(T, \prec)$, with $\prec$ a total reflection order, if and only if the order $t_{1} \prec \cdots \prec t_{k}$ is induced by some reduced expression of $w=t_{k} \cdots t_{1}$. In particular, if $W$ is finite, then we have $T=N(\omega)$, where $\omega$ is the longest element of $W$, and the total reflection orders are exactly the orders induced by the reduced expressions of $\omega$. In the following lemma we state a simple fact which will be useful in the following.

Lemma 4.1. Let $\left\{t_{1}, \ldots, t_{k}\right\} \subseteq T, s_{1}=t_{1}, s_{i}=t_{1} \cdots t_{i-1} t_{i} t_{i-1} \cdots t_{1}$ for $i=$ $2, \ldots, k$. Assume that $s_{1}, \ldots, s_{k} \in S$. Then $w=s_{1} \cdots s_{k}$ is a reduced expression and $N(w)=\left\{t_{1}, \ldots, t_{k}\right\}$.

Proof. Put $w_{i}=s_{1} \cdots s_{i}$, for $i=1, \ldots, k$; from the definitions it follows that $w_{i}=t_{i} \cdots t_{1}$. We prove by induction that $s_{1} \cdots s_{i}$ is reduced and $N\left(w_{i}\right)=$ $\left\{t_{1}, \ldots, t_{i}\right\}$. The case $i=1$ is obvious, so let $2 \leq i \leq k$. We have $N\left(w_{i}\right)=$ $N\left(w_{i-1}\right)+w_{i-1} N\left(s_{i}\right) w_{i-1}^{-1}=\left\{t_{1}, \ldots, t_{i-1}\right\}+\left\{t_{i-1} \cdots t_{1} s_{i} t_{1} \cdots t_{i-1}\right\}=\left\{t_{1}, \ldots, t_{i}\right\} ;$ in particular $s_{1} \cdots s_{i}$ is reduced.

We are now able to prove our result on finite Coxeter systems.

Theorem 4.2. Assume that $W$ is finite and let $\prec$ be a total order on $T$. If $\prec$ is $u$-stable, then it is a total reflection order.

Proof. We define inductively a sequence $\prec_{1} \stackrel{u}{\rightarrow} \cdots \prec_{i} \stackrel{u}{\rightarrow} \cdots$ setting

$$
\prec_{1}=\prec, \quad s_{i}=\min \left(S, \prec_{i}\right), \quad \prec_{i+1}=\prec_{i}^{s_{i}} .
$$

Then we set $n_{i}=\left|\left(s_{i} \preccurlyeq_{i}\right)\right|=\left|\left\{t \in T \mid s_{i} \preccurlyeq_{i} t\right\}\right|$. The sequence $\left(n_{i}\right)_{i \geq 1}$ is nonincreasing and positive; therefore for some $k \geq 1$ we have $n_{k}=n_{k+1}=\cdots$. For such 
a $k$, let $\left\{t_{1}, \ldots, t_{n_{k}}\right\}=\left(s_{k} \preccurlyeq_{k}\right)$ and $s_{k}=t_{1} \prec_{k} \cdots \prec_{k} t_{n_{k}}$. Then, by the choice of $k$, $\left\{t_{1}, \ldots, t_{n_{k}}\right\}$ satisfies the assumptions of Lemma 4.1 , so that $\left\{t_{1}, \ldots, t_{n_{k}}\right\}=N(w)$ for $w=t_{n_{k}} \cdots t_{1}$ and the order $t_{1} \prec_{k} \cdots \prec_{k} t_{n_{k}}$ is induced by a reduced expression of $w$. But, by our assumptions, $S \subseteq\left\{t_{1}, \ldots, t_{n_{k}}\right\}$; therefore $w$ is the longest element of $W$ and $N(w)=T$. It follows that $|T|=n_{1}=n_{2}=\cdots, s_{1}=\min \left(T, \prec_{1}\right)$, and $\prec_{1}=\prec$ is induced by a reduced expression of the longest element.

Now we study some consequences of $u$-stability for Coxeter groups in which each proper parabolic subgroup is finite.

Lemma 4.3. Let $\prec$ be a u-stable order on $T$ and $s \in S$ be the least simple root in $(T, \prec)$. Then, if $W$ is infinite, $(s \prec)$ is infinite.

Proof. Assume by contradiction that $(s \prec)$ is finite. We perform the same construction as that in the proof of Theorem 4.2; we consider the same sequence of $u$-stable orders $\prec_{i}$, the same sequence of non-negative integers $\left(n_{i}\right)$, and take $k$ such that $n_{k}=n_{k+1}=\cdots$. Then we have $S \subset(s \preccurlyeq)=N(w)$ for some $w \in W$, which is impossible since $W$ is infinite.

Theorem 4.4. Assume that each proper parabolic subgroup of $W$ is finite and let $\prec$ be a u-stable order on $T$. Then $(T, \prec)$ has a minimal element, which is a simple reflection. Moreover each finite initial section of $(T, \prec)$ is an initial section of some total reflection order.

Proof. We first prove that $\min (S, \prec)=\min (T, \prec)$. If $W$ is finite, the claim follows by Theorem 4.2 , so we assume that $W$ is infinite. We define inductively $\prec_{1}=\prec$, $s_{i}=\min \left(S, \prec_{i}\right)$, and $\prec_{i+1}=\prec_{i}{ }^{s_{i}}$. Then we consider the sequence $t_{1}=s_{1}, t_{i+1}=$ $s_{1} \cdots s_{i} s_{i+1} s_{i} \cdots s_{1}$, for $i \geq 1$. We shall prove that for each $i \geq 1$ we have $t_{i} \prec t_{i+1}$. In fact, by the definition of $s_{2}$, we have $t_{1}=s_{1} \prec s_{1} s_{2} s_{1}=t_{2}$. For $k \geq 2$, we assume inductively that $t_{1} \prec \cdots \prec t_{k}$. Then we have $s_{k} \prec_{k} s_{k} s_{k+1} s_{k}$. From the definitions $\left(s_{k} \prec_{k}\right)$ is equal to $\left(s_{k-1} \cdots s_{1}\right) \cdot\left(t_{k} \prec\right)$ plus a finite final section; if, by contradiction, $s_{k} s_{k+1} s_{k} \notin\left(s_{k-1} \cdots s_{1}\right) \cdot\left(t_{k} \prec\right)$, then $s_{k} s_{k+1} s_{k}$ is included in a finite final section of $\left(s_{k} \prec_{k}\right)$, which implies that $\left(s_{k+1} \prec_{k+1}\right)$ is finite, against Lemma 4.3. Thus $s_{k} s_{k+1} s_{k} \in\left(s_{1} \cdots s_{k-1}\right) \cdot\left(t_{k} \prec\right)$, which implies $t_{k+1} \in\left(t_{k} \prec\right)$, as claimed.

In particular, for each $k \geq 1$ the elements $t_{1}, \ldots, t_{k}$ are distinct and the sequence $t_{1}, \ldots, t_{k}$ satisfies the assumptions of Lemma 4.1 , so that $s_{1} \cdots s_{k}$ is a reduced expression. Since each proper parabolic subgroup of $W$ is finite, this implies that for each $s \in S$ and each $h \geq 1$ there exists a $k \geq h$ such that $s_{k}=s$.

Now we assume by contradiction that $\left(\prec_{1} s_{1}\right) \neq \emptyset$; then clearly $\left(\prec_{i} s_{i}\right) \neq \emptyset$ for each $i \geq 1$. Put $\ell_{i}=\min \left\{\ell(t) \mid t \prec_{i} s_{i}\right\}$; the sequence $\left(\ell_{i}\right)_{i \geq 1}$ is non increasing and greater than 2 , so that, for some $h \geq 1, \ell_{h}=\ell_{h+1}=\cdots$. Let $t \prec_{h} s_{h}$ be such that $\ell(t)=\ell_{h}$ and $s \in S$ be such that $\ell(s t s)<\ell(t)$. Then there exists a $k \geq h$ such that $s=s_{k}$; since $\left(\prec_{h} s_{h}\right) \subseteq\left(\prec_{k} s_{k}\right)$ we have $t \prec_{k} s_{k}$ and, by stability, $s t s=s_{k} t s_{k} \prec_{k} s_{k}$ : thus we have a contradiction, since $\ell(s t s)<\ell(t)=\ell_{k}$. Therefore we conclude that $s_{1}=\min (S, \prec)=\min (T, \prec)$, as claimed.

The second statement of the theorem follows easily from the above result. In fact, let $\left\{t_{1}, \ldots, t_{k}\right\}$ be an initial section of $T$ and set $s_{1}=t_{1}, s_{i}=t_{1} \cdots t_{i-1} t_{i} t_{i-1} \cdots t_{1}$ for each $i \in\{2, \ldots, k\}$. Then $s_{1}$ is simple, $s_{2}=t_{1} t_{2} t_{1}=\min \left(T, \prec^{t_{1}}\right)$ is simple and, inductively, $s_{i}$ is simple for each $i \in\{1, \ldots, k\}$. It follows, by Lemma 4.1, that $s_{1} \cdots s_{k}$ is reduced, and $\left\{t_{1}, \ldots, t_{k}\right\}=N\left(s_{1} \cdots s_{k}\right)$. Moreover the order $t_{1} \prec$ $\cdots \prec t_{k}$ is induced by the reduced expression $s_{1} \cdots s_{k}$. By [D2 2.11] we get the thesis. 
The results of this section have an analogous formulation for $l$-stable orders. Since a total order $\prec$ on $T$ is $l$-stable if and only if the reverse order is $u$-stable and since the reverse of a total reflection order is a total reflection order, we have that, for a finite Coxeter group, $l$-stable orders are total reflection orders. Moreover, if each proper parabolic subgroup of $W$ is finite, then the $l$-orders on $T$ have a maximum, which is a simple reflection, and the finite final sections of $l$-orders are the finite final sections of the total reflection orders.

\section{ACKNOWLEDGMENT}

I thank Francesco Brenti for many useful comments.

\section{REFERENCES}

[BB] A. Björner, F. Brenti, Combinatorics of Coxeter groups, to appear.

[B1] F. Brenti, Combinatorial expansion of Kazhdan-Lusztig polynomials, J. London Math Soc. 55 (1997), 448-472. MR 99a:05143

[B2] F. Brenti, Lattice paths and Kazhdan-Lusztig polynomials, J. Amer. Math. Soc. 11 (1998), 229-259. MR 98h:20072

[De] V.V. Deodhar, On the root system of a Coxeter group, Eins. Math. 32 (1986), 611-630. MR 83j:20052a

[D1] Dyer, M., Reflection subgroups of Coxeter systems, J. of Alg. 135 (1991), 57-73. MR 91j:20100

[D2] Dyer, M., Hecke algebras and shellings of Bruhat intervals, Comp. Math. 89 (1993), 91-115. MR 95c:20053

[H] J.E. Humphreys, Reflection groups and Coxeter groups, Cambridge University Press, 1990. MR 92h:20002

Dipartimento di Matematica Pura e Applicata, Università di Padova, Via Belzoni 7, 35131 PADOVA, ITALY

E-mail address: cellini@math.unipd.it 